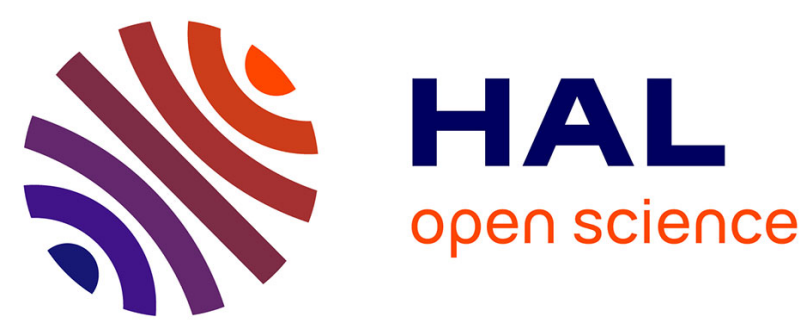

\title{
Space-Group Symmetries Generate Chaotic Fluid Advection in Crystalline Granular Media
}

Régis Turuban, D.R. Lester, Tanguy Le Borgne, Yves Méheust

\section{To cite this version:}

Régis Turuban, D.R. Lester, Tanguy Le Borgne, Yves Méheust. Space-Group Symmetries Generate Chaotic Fluid Advection in Crystalline Granular Media. Physical Review Letters, 2018, 120 (2), pp.024501. 10.1103/PhysRevLett.120.024501 . insu-01683973

\section{HAL Id: insu-01683973 \\ https://hal-insu.archives-ouvertes.fr/insu-01683973}

Submitted on 16 Dec 2019

HAL is a multi-disciplinary open access archive for the deposit and dissemination of scientific research documents, whether they are published or not. The documents may come from teaching and research institutions in France or abroad, or from public or private research centers.
L'archive ouverte pluridisciplinaire HAL, est destinée au dépôt et à la diffusion de documents scientifiques de niveau recherche, publiés ou non, émanant des établissements d'enseignement et de recherche français ou étrangers, des laboratoires publics ou privés. 


\title{
Space-Group Symmetries Generate Chaotic Fluid Advection in Crystalline Granular Media
}

\author{
R. Turuban, ${ }^{1}$ D. R. Lester, ${ }^{2, *}$ T. Le Borgne, ${ }^{1}$ and Y. Méheust ${ }^{1}$ \\ ${ }^{1}$ Geosciences Rennes, UMR 6118, Université de Rennes 1, CNRS, 35042 Rennes, France \\ ${ }^{2}$ School of Engineering, RMIT University, 3000 Melbourne, Australia
}

(Received 12 June 2017; published 9 January 2018)

\begin{abstract}
The classical connection between symmetry breaking and the onset of chaos in dynamical systems harks back to the seminal theory of Noether [Transp. Theory Statist. Phys. 1, 186 (1918)]. We study the Lagrangian kinematics of steady 3D Stokes flow through simple cubic and body-centered cubic (bcc) crystalline lattices of close-packed spheres, and uncover an important exception. While breaking of pointgroup symmetries is a necessary condition for chaotic mixing in both lattices, a further space-group (glide) symmetry of the bcc lattice generates a transition from globally regular to globally chaotic dynamics. This finding provides new insights into chaotic mixing in porous media and has significant implications for understanding the impact of symmetries upon generic dynamical systems.
\end{abstract}

DOI: 10.1103/PhysRevLett.120.024501

Since the foundational studies of dynamical systems, dating back to the seminal theory of Noether [1] regarding the connection between system symmetries and conserved quantities, symmetry breaking has become almost synonymous with the onset of chaotic dynamics [2]. This relationship has been repeatedly observed [3] over diverse systems spanning optical [4], celestial [5], quantum [6], and geophysical [7] applications. While a broad zoology of routes to chaos in dynamical systems have been documented [8], symmetry breaking overwhelmingly acts as a precursor to these transitions. We present a dynamical system which directly contradicts this notion, in that the presence of a space-group symmetry generates a transition from globally regular to globally chaotic dynamics. This hitherto unobserved phenomenon has significant implications for the understanding of chaos and bifurcations over a broad range of dynamical systems, as the importance of space-group symmetries has not been identified previously.

Specifically, we study the Lagrangian kinematics of steady Stokes flow through arrays of close-packed spheres, which represent the simplest embodiment of discrete (granular) porous media. These mixing mechanisms have received significant attention in the context of natural porous media [9-13], primarily driven by observations that classical dispersive concepts assuming well-mixed conditions fail to capture the mixing and reaction dynamics [14-18]. While recent studies have investigated fluid velocity and transport or mixing at the pore scale [19-23], the impact of pore scale structure upon mixing is an open question.

Recent studies [24-26] have also established that chaotic mixing is inherent to three-dimensional (3D) porous media for which the solid phase is continuous (i.e., not granular), such as open porous networks. While such chaotic mixing is driven by the topological complexity inherent to all porous media, it is unknown whether these concepts apply to discrete porous media as their topology is now contingent upon pointlike contacts between grains. Specifically, these cusp-shaped grain contacts render the fluid-solid boundary nonsmooth (i.e., it does not form a differentiable manifold), which invalidates the Poincaré-Hopf theorem central to the chaotic mixing mechanism.

To address these fundamental questions, we explore the Lagrangian kinematics of steady 3D Stokes flow in simple cubic (SC) and body-centered cubic (bcc) arrays of closepacked spheres. We study the mixing dynamics of the advection equation $\dot{\mathbf{x}}=\mathbf{v}(\mathbf{x})$, where "mixing" describes ergodicity of the (purely advective) Lagrangian kinematics rather than advection diffusion. Such chaotic fluid advection leads to accelerated scalar dispersion when coupled to molecular diffusion [25,27]. The $S O(3)$ rotational symmetry of the primary particles means that the mixing dynamics are solely governed by the basic lattice structure and relative mean flow orientation. As for generic dynamical systems, symmetries play a key role in controlling the Lagrangian topology [28-32]. We aim at understanding the link between mixing dynamics, flow orientation, and the underlying lattice symmetries.

The SC and bcc sphere arrays can be represented by the base vectors $\mathbf{s}^{(i)}, i=1: 3$ of their primitive cells shown in Fig. 1(a). The close-packed, unit radii spheres are centered at positions $\mathbf{r}=\mathbf{r}_{0}+\sum_{i=1}^{3} m_{i} \mathbf{s}^{(i)}$ with $m_{i}=0, \pm 1, \ldots$, and $\mathbf{s}^{(1)}=2(1,0,0), \mathbf{s}^{(1)}=2 / \sqrt{3}(1,1,-1)$ for the SC and bcc lattices, respectively, where $\mathbf{s}^{(2)}, \mathbf{s}^{(3)}$ are given by cyclical permutations of these vectors. The unit cell lengths of the SC and bcc lattices are $\ell=2,4 / \sqrt{3}$, respectively, and $\mathbf{r}_{0}=(-\ell / 2,-\ell / 2,-\ell / 2)$. As the octahedral bcc primitive cell is the dual of the cubic SC primitive cell, the SC and 


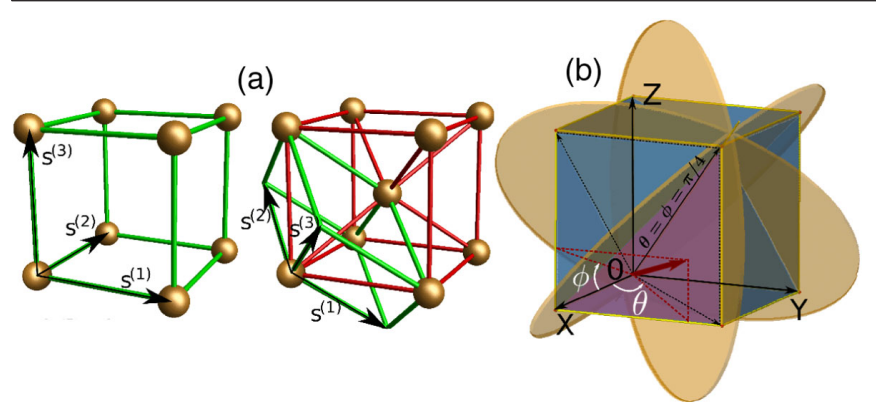

FIG. 1. (a) Primitive (green) and unit (red) cells for the SC (left) and bcc (right) packings (spheres are shrunk for visualization). (b) Selected $S_{2}^{m}$ reflection symmetry planes (yellow discs) of the SC and bcc unit cells $\{[1-10],[10-1],[01-1]\}$ which intersect along $(\theta, \phi)=(\pi / 4, \pi / 4)$. The purple region indicates the flow parameter space $\mathcal{H}:\left(\phi_{f}, \theta_{f}\right)=[0, \pi / 4] \times[0, \pi / 4]$. Adapted from [33].

bcc lattices share the same $(\mathrm{m} 3 \mathrm{~m})$ symmetry point group, which consists of two sets of equivalent reflection symmetries: $S_{1}^{m}=\{[100],[010],[001]\}$ and $S_{2}^{m}=\{[110],[101]$, [011], $[1-10],[10-1],[01-1]\}$, some of which are shown in Fig. 1(b). We denote the $S_{1}^{m}$ symmetry planes on the unit cell midplanes (faces) as $L_{i}\left(L_{i}^{\prime}\right)$, with $i \in\{X, Y, Z\}$. The normal vectors of the 9 reflection symmetry planes associated with $S_{1}^{m}, S_{2}^{m}$ can be expressed in spherical coordinates as $(\theta, \phi) \in(n \pi / 4, m \pi / 4)$ for all 9 permutations of $n, m \in\{-1,0,1\}$, where $(\theta, \phi)=(0,0)$, aligns with $\hat{\mathbf{e}}_{X}$ [Fig. 1(b)]. The mean flow orientation is denoted by the spherical coordinates $\left(\theta_{f}, \phi_{f}\right)$. For both lattices the $S_{1}^{m}$ and $S_{2}^{m}$ point group symmetries reduce the set of unique flow orientations $\left(\theta_{f}, \phi_{f}\right)$ to the parameter space $\mathcal{H}:\left(\theta_{f}, \phi_{f}\right) \in[0, \pi / 4] \times[0, \pi / 4]$.

To study the Lagrangian dynamics in the SC and bcc lattices, we compute the steady 3D Stokes velocity field $\mathbf{v}(\mathbf{x})$ over $\mathcal{H}$ to high precision (rms error $10^{-14}$ ) over the fluid domain $\mathcal{D}$ within each lattice primitive cell. No-slip conditions are imposed on the sphere surfaces $\partial \mathcal{D}$, and periodic conditions on the cell faces. To quantify the magnitude of fluid deformation per unit cell length $\ell$ traversed in the longitudinal direction, we calculate the infinite-time Lyapunov exponent $\lambda$ as

$$
\lambda \equiv \lim _{t \rightarrow \infty} \frac{\ell}{2 s(t)} \ln [\nu(t)]
$$

where $\nu(t)$ is the largest eigenvalue of the Cauchy-Green tensor $\mathbf{C}(\mathbf{X}, t)=\mathbf{F}^{\top} \cdot \mathbf{F}, \mathbf{F}$ the deformation gradient tensor, $s(t) \equiv \mathbf{q} \cdot(\mathbf{x}(t)-\mathbf{x}(0)) /|\mathbf{q}|$ the longitudinal distance travelled at time $t$, and $\mathbf{q}$ the mean flow vector. To improve convergence, Eq. (1) is computed in streamline coordinates $[34,35]$ from a random set of initial positions $\mathbf{X}$; hence, $\lambda$ represents an average over all distinct Lagrangian topologies of the flow. The distribution of $\lambda$ over $\mathcal{H}$ for the two lattices is shown in Fig. 2(c).

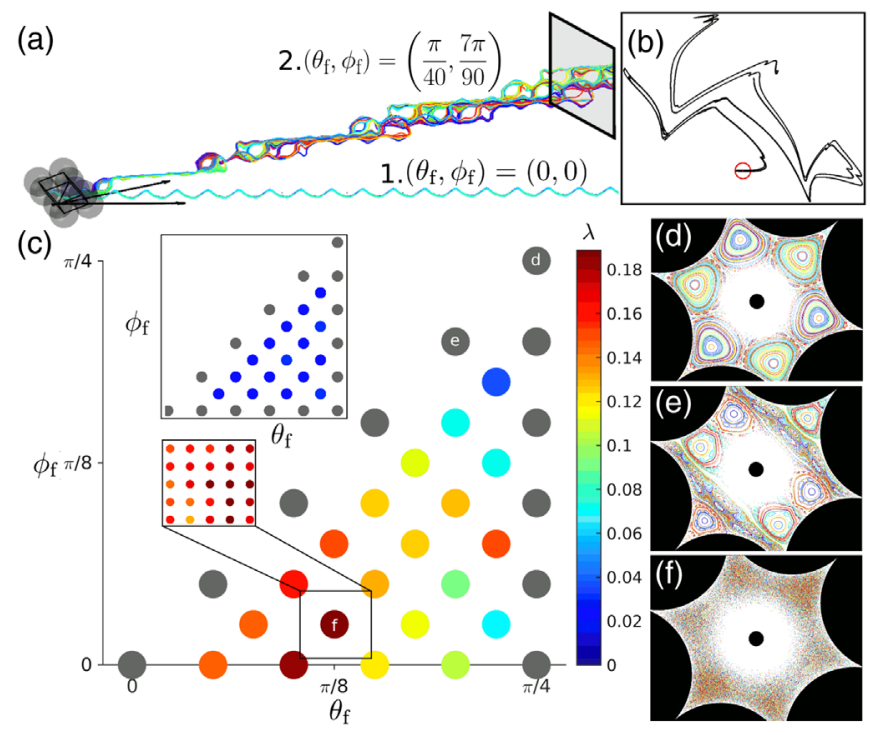

FIG. 2. (a) Particle trajectories for two flow orientations in the BCC lattice: $1\left(\theta_{f}, \phi_{f}\right)=(0,0), 2\left(\theta_{f}, \phi_{f}\right)=(\pi / 40,7 \pi / 90)$, illustrating the sharp transition from regular flow to strong chaotic mixing under small changes in the mean flow orientation. (b) Intersection (black line) of a continuously injected material line with the window shown in (a) for flow orientation 2; the red circle indicates the upstream injection line (see video Anim1 [36]). (c) Distribution of the Lyapunov exponent $\lambda$ over $\mathcal{H}$ for BCC (main figure) and SC (upper inset) packings, where gray denotes regular dynamics $(\lambda=0)$ due to alignment of the mean flow with the normal to a reflection symmetry plane. (d)-(f) Poincaré sections [normal to $(1,1,1)$ ] for the BCC lattice, for flow orientations $\left(\theta_{f}, \phi_{f}\right)$ : (d) $(\pi / 4, \pi / 4)$, (e) $(\pi / 5, \pi / 5)$, (f) $(\pi / 8, \pi / 40)$, with corresponding $\lambda$ shown in (c).

The orientation of the mean flow with respect to the $S_{m}^{1}$, $S_{m}^{2}$ reflection symmetries is fundamental to Lagrangian dynamics. Figure 2(a) depicts a sharp transition from regular, periodic streamlines when the flow is normal to a $S_{1}^{m}$ reflection symmetry, to strong chaotic mixing for a weak breaking of this symmetry [Fig. 2(b)]. The $S_{m}^{1}, S_{m}^{2}$ reflection symmetries also explain the completely regular Lagrangian dynamics $(\lambda=0)$ observed for both lattices along the transects $\theta_{\mathrm{f}}=\pi / 4$ and $\theta_{\mathrm{f}}=\phi_{\mathrm{f}}$ in $\mathcal{H}$, as reflected by the nested KAM tori shown in Figs. 2(d), 2(e).

In addition to these reflection symmetries, the bcc lattice also possesses a set of space group glide symmetries consisting of a translation and reflection, e.g.,

$\mathcal{G}_{Z}^{ \pm}:(X, Y, Z) \mapsto(X+\ell / 2, Y+\ell / 2,-Z \pm \ell / 2)$,

with similar glide symmetries for $X, Y$ reflections. These glide symmetries do not occur in the SC lattice due to absence of a central sphere in the unit cell. In the bcc lattice, for mean flows oriented parallel to the planes $G_{Z}^{ \pm}: Z=$ $\pm \ell / 4$ [Fig. 3(a)], a series of material surfaces (consisting of streamlines) $\mathcal{M}_{Z}^{ \pm}$satisfies the glide symmetries $\mathcal{G}_{Z}^{ \pm}$. As shall be shown, these play a critical role in the generation of chaotic mixing. 


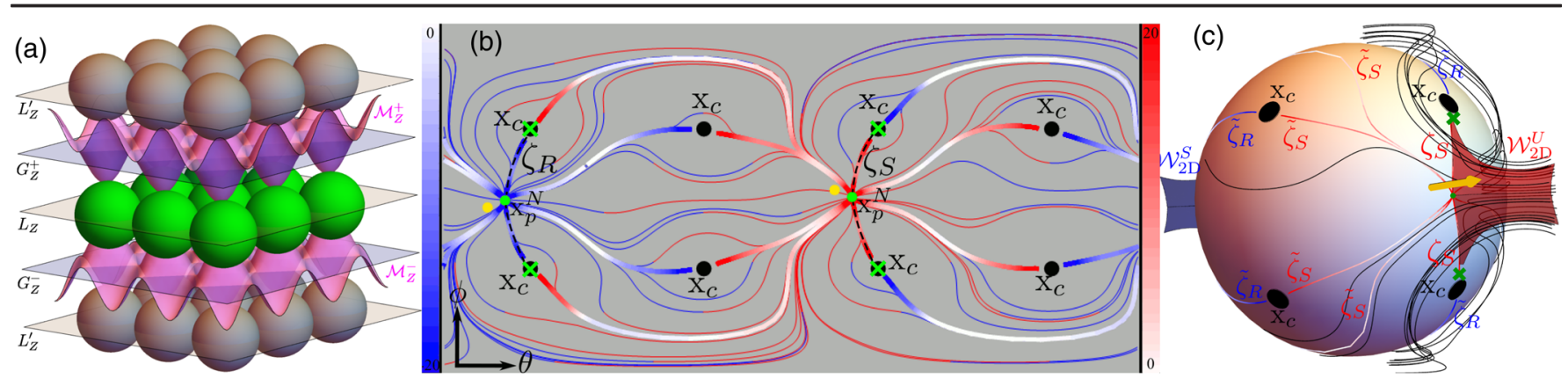

FIG. 3. (a) Vertically exploded view of sphere layers in the BCC lattice (with color convention identical to that in Fig. 5), $L_{Z}, L_{Z}^{\prime}$ reflection (gray) and $G_{Z}^{ \pm}$glide (blue) symmetry planes and material surfaces $\mathcal{M}_{Z}^{ \pm}$(pink) for $\phi_{\mathrm{f}}=0$. (b) Forward (red) and backward (blue) skin friction lines (thin) in spherical coordinates $(\theta, \phi)$ over the sphere surface $\partial \mathcal{D}$ in the BCC lattice for $\left(\theta_{\mathrm{f}}, \phi_{\mathrm{f}}\right)=(\pi / 8, \pi / 40)$, with mean flow orientation (yellow dots), node $\mathbf{x}_{p}^{N}$ (green dots), saddle $\mathbf{x}_{p}^{S}$ (green crosses), and contact $\mathbf{x}_{c}$ points (black discs) shown. The separation (red) and reattachment (blue) critical lines $\zeta$ (thick, black dashed lines) and degenerate critical lines $\tilde{\zeta}$ (thick lines) are colored according to $(\nabla \cdot \mathbf{u}) /\|\mathbf{u}\|$ (left/right color bars). (c) 3D view of the same skin friction field, showing fluid streamlines (black), stable (blue), and unstable (red) 2D manifolds emanating from critical lines $\zeta$.

From the flow computations over $\mathcal{H}$, we find that chaotic mixing within the bcc lattice is significantly stronger $(\lambda \in[0,0.19])$ than within the SC lattice $(\lambda \in[0,0.02])$, and exhibits a rich distribution of Lagrangian topologies over $\mathcal{H}$ [Fig. 2(c)], as illustrated by Figs. 2(d)-2(f). These differences are highlighted by the different behaviors along the $\phi_{\mathrm{f}}=0$ transect in $\mathcal{H}$, for which we observe strong global chaotic mixing in the bcc lattice, whereas for the SC lattice the flow is completely regular.

To explain these observations, we investigate the mechanisms of chaotic mixing in discrete porous media. As discussed below they are fundamentally different from those discovered in continuous porous media [24]. Under certain conditions, interaction of flow with the sphere surfaces $\partial \mathcal{D}$ generates $2 \mathrm{D}$ hyperbolic separation and reattachment surfaces [Fig. 3(c)]. These, respectively termed 2D unstable $\mathcal{W}_{2 \mathrm{D}}^{\mathrm{U}}$ and stable $\mathcal{W}_{2 \mathrm{D}}^{\mathrm{S}}$ manifolds, respectively repel or attract streamlines exponentially. Chaotic mixing then results if these (un)stable manifolds intersect transversely in the fluid bulk [see Fig. 4(c) and 3D animation 'Anim2' in Ref. [36]), forming a heteroclinic tangle [24,37].

We first consider the conditions for the generation of such manifolds. These are tied to the existence of saddle

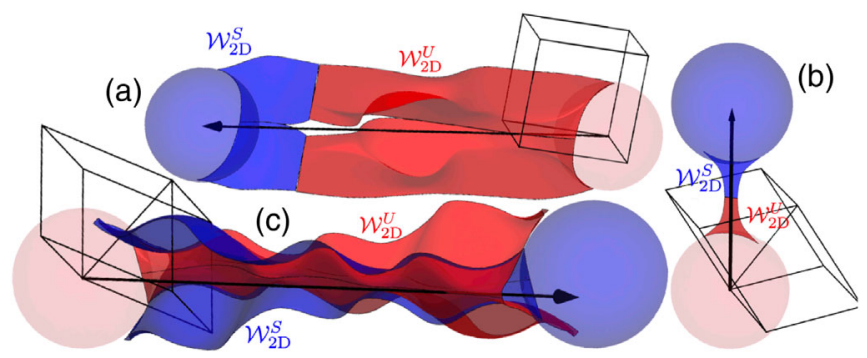

FIG. 4. Smooth heteroclinic connections for (a) the SC lattice with $\left(\theta_{f}, \phi_{f}\right)=(3 \pi / 20,0)$ (see also 3D animation Anim3 in Ref. [36]) and (b) the bcc lattice with $\left(\theta_{f}, \phi_{f}\right)=(\pi / 4,0)$. (c) Transverse heteroclinic intersection for the bcc lattice with $\left(\theta_{\mathrm{f}}, \phi_{\mathrm{f}}\right)=(\pi / 20,0)$ (see also 3D animation Anim2 in Ref. [36]). points in the skin friction field $\mathbf{u}\left(x_{1}, x_{2}\right) \equiv \partial \mathbf{v} /\left.\partial x_{3}\right|_{x_{3}=0}$ over $\partial \mathcal{D}$ [Fig. 3(b)], where $\left(x_{1}, x_{2}\right)$ and $x_{3}$, respectively, are tangent and normal to $\partial \mathcal{D}$. Nondegenerate critical points $\mathbf{x}_{p}$ of the skin friction field [i.e., $\mathbf{u}\left(\mathbf{x}_{p}\right)=\mathbf{0}$, $\mathcal{A} \equiv \partial \mathbf{u} /\left.\partial \mathbf{x}\right|_{\mathbf{x}_{p}} \neq \mathbf{0}$ ] can be classified as nodes $\mathbf{x}_{p}^{N}$, saddles $\mathbf{x}_{p}^{S}$, or centers $\mathbf{x}_{p}^{C}$, based on whether the real parts of the eigenvalues of $\mathcal{A}$ have the same sign, different signs, or are both zero. Nondegenerate saddle-node connections in $\mathbf{u}$ form critical lines on the sphere surface; vector field lines of $\mathbf{u}$ that act as strongly hyperbolic [38] attractors or repellers. Because of mass conservation, separation $\zeta_{S}\left(\nabla_{\perp} \cdot \mathbf{u}<0\right)$ and reattachment $\zeta_{R}(\nabla \perp \cdot \mathbf{u}>0)$ critical lines, respectively, generate 2D hyperbolic unstable and stable manifolds which emanate from the sphere surface into the pore space.

In all continuous porous media the topological complexity inherent to the geometry generates a large number density of nondegenerate saddle points in the skin friction field [24], which then guarantees the formation of 2D (un) stable manifolds. However, for discrete porous media that complex topology is no longer sufficient to guarantee the formation of 2D (un)stable manifolds, due to the nonsmooth nature of the fluid-solid boundary, which renders saddle points local to grain contacts as degenerate $[39,40]$.

Despite this, we consistently observe formation of $2 \mathrm{D}$ (un)stable manifolds throughout $\mathcal{H}$ for the $\mathrm{SC}$ and bcc lattices [Fig. 3(a), 4(a)-4(c)]. Indeed their formation is rendered possible in discrete media by bifurcations of the skin friction field under steady flow (allowing this field to admit nondegenerate saddle points, see Fig. 3). In this sense fluid mixing differs markedly between continuous and discrete porous media, as topological complexity alone is insufficient to generate chaotic mixing in discrete porous media. This explains the weaker mixing observed in granular media $(\langle\lambda\rangle \approx 0.073$ over the $\mathrm{SC}$ and bcc lattices) with respect to open porous networks $(\langle\lambda\rangle \approx 0.118$ [24]).

Given formation of 2D (un)stable manifolds, chaotic mixing then hinges upon these forming a transverse 
heteroclinic connection. To understand the markedly different Lagrangian kinematics between the bcc and SC lattices, we consider the set of flow orientations $\phi_{\mathrm{f}}=0$, for which the SC lattice exhibits globally regular dynamics while the bcc lattice shows global chaotic mixing. For $\phi_{f}=0$ the flow is parallel to the reflection symmetry planes $L_{Z}, L_{Z}^{\prime}$ [Fig. 3(a)], so $L_{Z}$ is a material surface, which forces the streamlines $\alpha^{S}, \alpha^{U}$ formed by intersection of this plane with $\mathcal{W}_{2 \mathrm{D}}^{S}, \mathcal{W}_{2 \mathrm{D}}^{U}$ to connect smoothly [Fig. 5(a)]. However, transverse connections are possible away from $L_{Z}$, depending upon the curvature of the manifolds.

This is shown in Fig. 5(b), where $L_{f}^{\prime}$ is the symmetry plane normal to the mean flow direction and midway between the spheres $S_{0}, S_{1}$ [Fig. 5(a)]. The (un)stable manifolds attached to these spheres are doubly symmetric in $L_{f}^{\prime}$ due to the reflection symmetries of $L_{X}, L_{Y}, L_{Z}$, and the time-reversal symmetry between $\mathcal{W}_{2 \mathrm{D}}^{S}$ and $\mathcal{W}_{2 \mathrm{D}}^{U}$. As $L_{Z}$, $L_{Z}^{\prime}$ are symmetry planes, $\mathcal{W}_{2 \mathrm{D}}^{S}$ and $\mathcal{W}_{2 \mathrm{D}}^{U}$ can only intersect these normally for both $\mathrm{SC}$ and bcc lattices. For the SC

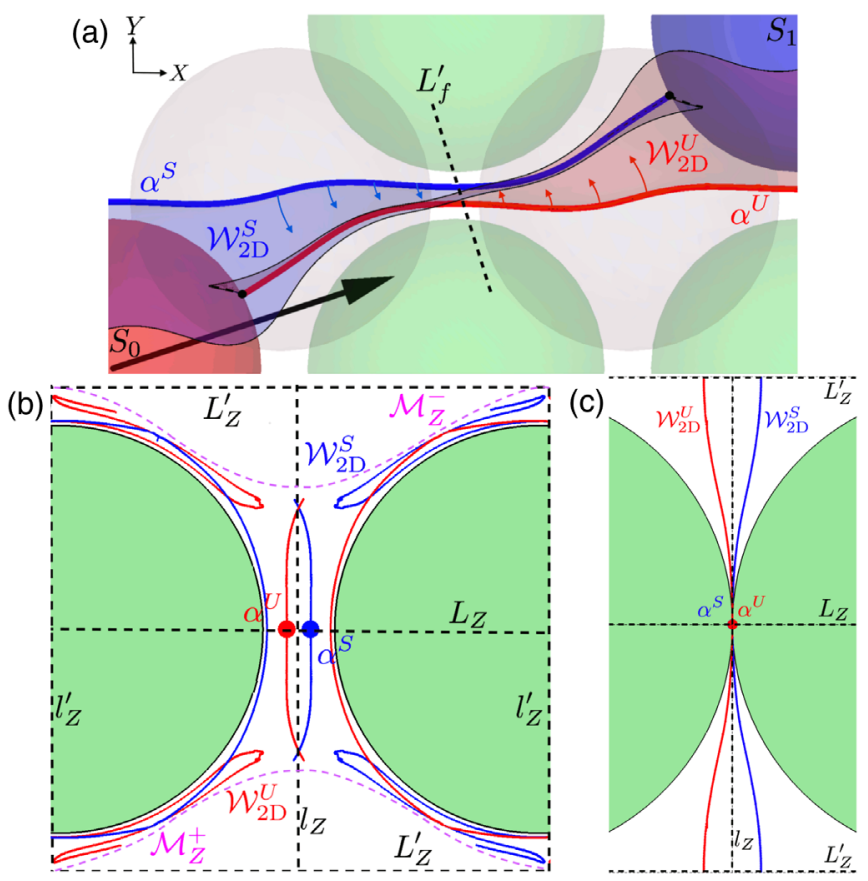

FIG. 5. (a) Projection in the $Z$ direction of spheres in the $L_{Z}$ (green) and $L_{Z}^{\prime}$ (gray) symmetry planes of the bcc lattice for $\left(\theta_{\mathrm{f}}, \phi_{\mathrm{f}}\right)=(\pi / 10,0)$. The $S_{0}$ (red) and $S_{1}$ (blue) spheres in $L_{Z}$ interact via their respective stable $\mathcal{W}_{2 \mathrm{D}}^{S}$ (blue) and unstable $\mathcal{W}_{2 \mathrm{D}}^{U}$ (red) manifolds and associated streamlines $\alpha^{S}, \alpha^{U}$ (thick lines) in $L_{Z}$. (b),(c) Projection of spheres and manifolds in $L_{f}^{\prime}$ plane in the bcc and SC lattices respectively. (b) Concavity of the (un)stable manifolds [as indicated by the red and blue arrows in (a)] results in a transverse heteroclinic connection away from $L_{Z}, L_{Z}^{\prime}$ (see 3D animation Anim2 [36]). The pink dotted lines denote the material surfaces $\mathcal{M}_{Z}^{ \pm}$, and the manifolds wrapping around the spheres are forward or backward iterates [35] of the manifolds shown in the center. (c) For the SC lattice, convexity of the manifolds about $l_{Z}$ results in smooth connections for all $\theta_{\mathrm{f}}$ along $\phi_{\mathrm{f}}=0$. lattice, this leads to uniformly smooth connections between (un)stable manifolds [Fig. 5(c)] for all $\phi_{f}=0$, as there is no mechanism to destroy convexity of the manifolds about the symmetry plane $l_{Z}$ of the lattice normal to $L_{f}^{\prime}$. This behavior explains the globally regular Lagrangian dynamics $(\lambda=0)$ observed for the SC lattice along $\phi_{f}=0$.

Conversely, such crossing of the symmetry planes $L_{Z}^{\prime}$ by $\mathcal{W}_{2 \mathrm{D}}^{S}, \mathcal{W}_{2 \mathrm{D}}^{U}$ is not possible in the BCC lattice due to presence of the glide symmetries $\mathcal{G}_{Z}^{ \pm}$. For $\phi_{\mathrm{f}}=0$, they give rise to the set of "egg tray"-shaped material surfaces $\mathcal{M}_{Z}^{ \pm}$ between horizontal sphere layers [Fig. 3(a)]. These surfaces contain the contact points between spheres and partition the fluid domain into isolated layers between the $\mathcal{M}_{Z}^{ \pm}$ surfaces and the $L_{Z}, L_{Z}^{\prime}$ symmetry planes. Unlike the $L_{Z}$, $L_{Z}^{\prime}$ symmetry planes, the translational component of these glide symmetries $\mathcal{G}_{Z}^{ \pm}$forbids transverse crossing of $\mathcal{M}_{Z}^{ \pm}$by the 2D (un)stable manifolds. This confines the manifolds between $\mathcal{M}_{Z}^{+}, \mathcal{M}_{Z}^{-}$, resulting in the heteroclinic tangle shown in Fig. 5(b). Forward or backward iterates [35] of these manifolds shown in Fig. 5(b) illustrate the development of the heteroclinic tangle and the formation of chaotic mixing.

Thus, the additional glide symmetries are directly responsible for the vastly different Lagrangian kinematics between the SC and bcc lattices along the transect $\phi_{f}=0$. As the other boundaries $\left(\theta_{f}=\phi_{f}, \theta_{f}=\pi / 4\right)$ of $\mathcal{H}$ involve globally regular flows for each lattice, the dynamics along $\phi_{f}=0$ inform the mixing dynamics over $\mathcal{H}$ more broadly. While the Lyapunov exponent for the bcc lattice can be large along $\phi_{\mathrm{f}}=0$, mixing is isolated to material regions bound between $L_{Z}, L_{Z}^{\prime}$ and $\mathcal{M}_{Z}^{ \pm}$[Fig. 5(b)], leading to zero net transverse dispersion. A small perturbation in $\phi_{\mathrm{f}}$ breaks the associated symmetries, resulting in global chaos and transverse dispersion [Fig. 2(b)]. Indeed, the strongest mixing case computed within $\mathcal{H}$ for the bcc lattice lies just off the $\phi_{\mathrm{f}}=0$ transect, near the maximum value of $\lambda$ along $\phi_{f}=0$ [Figs. 2(c), 2(f)]. Conversely, the SC lattice only exhibits weak mixing throughout $\mathcal{H}$, which we attribute to the regular dynamics along all boundaries of $\mathcal{H}$.

In random granular or discrete porous media glide symmetries do not exist, but the basic mechanisms of flow separation or reattachment and transverse manifold intersections will persist. There is significant indirect evidence of chaotic mixing in random discrete media [see, e.g., the entangled streamlines in Fig. 2 in Ref. [41], Fig. 3 in Ref. [21], and Fig. 8(a) in Ref. [42]]. Investigation of the underlying mechanisms is the subject of current work.

We have used the skin friction topology, numerical calculation of the manifolds' geometry, and symmetry analysis of bcc and SC sphere lattices, to elucidate the mechanisms governing chaotic mixing and the Lyapunov exponent distribution over the flow orientation space. We find (i) that the pointwise grain contacts fundamentally alter the nature of chaotic advection in discrete porous media, 
leading to weaker mixing than in continuous media, and (ii) that space group glide symmetries can directly generate chaotic dynamics, which explains the vastly different mixing dynamics between the bcc and SC lattices. This hitherto unreported impact of space-group symmetries upon chaotic dynamics contradict classical observations [1] that symmetry breaking is linked with the formation of transverse manifold connections and chaos, both within studies of chaotic mixing [28-31] and dynamical systems in general [2]. This apparent contradiction arises from the fact that prior studies of symmetries and chaos have focused upon point-group symmetries whereas glide symmetries are space-group symmetries, which points to an extension of existent field-invariant symmetry analyses (e.g., Refs. [28,29]) to develop a broader framework for the impact of space-group symmetries upon generic dynamical systems.

The authors acknowledge the support of the European Research Council (ERC) through the consolidator grant ReactiveFronts (648377) and of the Agence Nationale de la Recherche (ANR) through the project 'Subsurface Mixing and Reaction' (ANR-14-CE04-0003).

*daniel.lester@rmit.edu.au

[1] E. Noether, Transp. Theory Statist. Phys. 1, 186 (1918).

[2] K. G. Szabó and T. Tél, J. Stat. Phys. 54, 925 (1989).

[3] Over 22000 citations in Google scholar.

[4] X.-Y. Lü, H. Jing, J.-Y. Ma, and Y. Wu, Phys. Rev. Lett. 114, 253601 (2015).

[5] R. L. Jennings and N. O. Weiss, Mon. Not. R. Astron. Soc. 252, 249 (1991).

[6] R. Blümel and U. Smilansky, Phys. Rev. Lett. 69, 217 (1992).

[7] L. R. M. Maas, J. Fluid Mech. 437, 13 (2001).

[8] L. O. Chua, C. W. Wu, A. Huang, and G.-Q. Zhong, IEEE Trans. Circuits Syst. I 40, 732 (1993).

[9] N. Kleinfelter, M. Moroni, and J. H. Cushman, Phys. Rev. E 72, 056306 (2005).

[10] B. Jha, L. Cueto-Felgueroso, and R. Juanes, Phys. Rev. Lett. 106, 194502 (2011).

[11] F. P. J. de Barros, M. Dentz, J. Koch, and W. Nowak, Geophys. Res. Lett. 39 (2012).

[12] T. Le Borgne, M. Dentz, and E. Villermaux, Phys. Rev. Lett. 110, 204501 (2013).

[13] Y. Ye, G. Chiogna, O. A. Cirpka, P. Grathwohl, and M. Rolle, Phys. Rev. Lett. 115, 194502 (2015).

[14] C. M. Gramling, C. F. Harvey, and L. C. Meigs, Environ. Sci. Technol. 36, 2508 (2002).

[15] A. M. Tartakovsky, D. M. Tartakovsky, and P. Meakin, Phys. Rev. Lett. 101, 044502 (2008).

[16] I. Battiato, D. M. Tartakovsky, A. M. Tartakovsky, and T. Scheibe, Adv. Water Resour. 32, 1664 (2009).
[17] M. Dentz, T. LeBorgne, A. Englert, and B. Bijeljic, J. Contam. Hydrol. 120-121, 1 (2011).

[18] P. de Anna, J. Jimenez-Martinez, H. Tabuteau, R. Turuban, T. L. Borgne, M. Derrien, and Y. Meheust, Environ. Sci. Technol. 48, 508 (2014).

[19] B. Bijeljic, P. Mostaghimi, and M. J. Blunt, Phys. Rev. Lett. 107, 204502 (2011)

[20] P. K. Kang, P. de Anna, J. P. Nunes, B. Bijeljic, M. J. Blunt, and R. Juanes, Geophys. Res. Lett. 41, 6184 (2014).

[21] M. Holzner, V. L. Morales, M. Willmann, and M. Dentz, Phys. Rev. E 92, 013015 (2015).

[22] J. Jiménez-Martínez, P. de Anna, H. Tabuteau, R. Turuban, T. L. Borgne, and Y. Méheust, Geophys. Res. Lett. 42, 5316 (2015).

[23] J. Jiménez-Martínez, T. Le Borgne, H. Tabuteau, and Y. Méheust, Water Resour. Res. 53, 1457 (2017).

[24] D. R. Lester, G. Metcalfe, and M. G. Trefry, Phys. Rev. Lett. 111, 174101 (2013).

[25] D. R. Lester, M. Dentz, and T. Le Borgne, J. Fluid Mech. 803, 144 (2016).

[26] D. Lester, M. Trefry, and G. Metcalfe, Adv. Water Resour. 97, 175 (2016).

[27] J. M. Ottino, The Kinematics of Mixing: Stretching, Chaos, and Transport (Cambridge University Press, Cambridge, England, 1989).

[28] I. Mezić and S. Wiggins, J. Nonlinear Sci. 4, 157 (1994).

[29] G. Haller and I. Mezic, Nonlinearity 11, 319 (1998).

[30] A. N. Yannacopoulos, I. Mezić, G. Rowlands, and G. P. King, Phys. Rev. E 57, 482 (1998).

[31] J. G. Franjione, C.-W. Leong, and J. M. Ottino, Phys. Fluids A 1, 1772 (1989).

[32] D. R. Lester, M. Rudman, G. Metcalfe, M. G. Trefry, A. Ord, and B. Hobbs, Phys. Rev. E 81, 046319 (2010).

[33] http://symmetry.otterbein.edu.html (2017).

[34] D. R. Lester, M. Dentz, T. L. Borgne, and F. P. J. D. Barros, J. Fluid Mech. (to be published).

[35] See Supplemental Material at http://link.aps.org/ supplemental/10.1103/PhysRevLett.120.024501 for consists of two different sections. The first section details the procedure used to calculate the lyapunov exponent. The second section describes how the forward and backward iterates of the manifolds shown in Fig. 5(b) are calculated.

[36] https://reactivefronts-erc.univ-rennes1.fr/data/supanimations-turuban-et-al-prl-in-press/ (2017).

[37] R. S. MacKay, Philos. Trans. R. Soc., A 359, 1479 (2001).

[38] A. Surana, O. Grunberg, and G. Haller, J. Fluid Mech. 564, 57 (2006).

[39] M. Brøns and J. N. Hartnack, Phys. Fluids 11, 314 (1999).

[40] R. Turuban, D. R. Lester, T. L. Borgne, and Y. Méheust (unpublished).

[41] J. Katagiri, H. Saomoto, and M. Utsuno, Vadose Zone J. 14 (2015).

[42] B. P. Muljadi, M. J. Blunt, A. Q. Raeini, and B. Bijeljic, Adv. Water Resour. 95, 329 (2016). 